\title{
The modified metric for self-organization wireless MESH networks
}

\author{
Andrzej Paszkiewicz ${ }^{1, *}$, and Przemystaw Zapała ${ }^{2}$ \\ ${ }^{1}$ Department of Complex Systems, The Faculty of Electrical and Computer Engineering, Rzeszow \\ University of Technology, al. Powstańców Warszawy 12, 35-959 Rzeszów, Poland \\ ${ }^{2}$ Rzeszów, Poland
}

\begin{abstract}
This paper concerns the modified metrics for MESH wireless networks. This metric is important when a specific data transmission path is being chosen. The parameters regarding the connection quality and overload are introduced in it. Therefore, the proposed metric takes a closer look at changing conditions of the wireless environment and contributes to a better self-organization functioning of this type of network. Its importance increases for networks characterized by the transmission of converged traffic. The paper also presents the results of a simulation showing the dependence of metric values on transmission parameters for selected wireless transmission technologies.
\end{abstract}

\section{Introduction}

Self-organization is a known term and increasingly popular in relation to broadly understood computer technologies including computer networks [1-4]. First references to this term were associated with the natural systems, mainly biology [5]. Scientists, observing living organisms, their behavior and reactions to changing conditions (i.e. environmental) create algorithms and self-organizing mechanisms implemented in artificial systems (i.e. telephone networks).

A very good example of network mechanism that adjusts to the dynamic changes of the conditions and transmission parameters are routing algorithms. These mechanisms make decisions to choose the 'best path' for data transmission based on information they have and which is updated. This decision is made individually by each of the nodes. Thus, such a structure resembles a system of independent agents cooperating to achieve a common goal, and their impact on the entire structure (the community) is smaller or larger. It is obvious that computer networks, especially wired ones, to a small extent of their functionality correspond to the definition of multi-agent systems. Whereas wireless networks are very good example of multi-agent systems, especially sensor networks or wireless MESH networks.

MESH networks are a collection of independent nodes, which provide access to the network over a wider area without the need of supplying wired links $[6,7]$. This solution

\footnotetext{
* Corresponding author: andrzejp@prz.edu.pl
} 
allows to cover the large area in which the use of alternative technology is not always possible. To ensure communication in the whole network infrastructure, individual nodes are making independent decisions. They are made within the network without the central unit. Therefore, this structure corresponds to the network consisting of independent agents and fits into a concept of self-organization systems, where individual nodes can connect and disconnect at any time, and the network as a whole system still functions as a coherent environment. The system works according to the determined guidelines and principles to which agents have to adapt, so that actions of all agents are coherent.

Of course, the self-organizing system can be considered from the point of view of different functionalities [8]. In this paper we will focus on the selection of the communication link from the point of view of each node. This choice will be based on the metric that adapts to changing conditions of transmission. This metric directly decides about specific transmission path based on current information. Routing metrics, especially in wired networks, are usually based on simple parameters associated with distance or connection bandwidth. Taking the stability of environment in wired networks into consideration such assumptions may work. However, in wireless networks, especially in the 802.11 technology, there are many transmission interferences and restrictions. Furthermore, the dynamics of these changes is difficult to predict, therefore, the metric should adapt to them.

The fundamental elements responsible for the routes optimization in this protocol are connection metrics, which are also analyzed in this paper. In order to improve the route selection mechanism, the existing metrics were modified.

\section{Fundamentals of MESH networks}

Nowadays, wireless MESH networks are widely used including provision of communication for fixed and temporary infrastructure [6]. The examples of temporary infrastructure can be big celebrations, sport events, congresses organized in a large area, or providing communication in crisis situations. Excellent examples for permanent infrastructure are parks and university campuses. In these places it is not always possible to bring logical infrastructure (i.e. protection of monuments and wildlife) or the costs are too high in the case of fixed infrastructure.

The MESH network consists of the access points that communicate with each other creating a certain form of self-organizing system Fig.1. In this system, MESH nodes fulfill the role of agents by collecting information from their environment. MESH network is comparable to social model of the multi-agent system.

The role of nodes known as MESH portal is to be a gateway between wired and wireless network. There can be many MESH portals in the given network infrastructure. Thus, reliability is increased and additional load balancing mechanisms can be provided. However, the nodes known as MESH point ensure preservation of the wireless path between MESH nodes. In addition, they can ensure network access for wireless end users. Of course, exactly how this type of network works is described in the 802.11s standard, as well as in numerous publications [7].

Routing protocols have an important role that concerns all types of computer networks. The MESH wireless networks mainly use the Hybrid Wireless Mesh Protocol (HWMP). Other protocols are also possible. The HWMP is a hybrid protocol that is based on a reactive protocol Ad hoc On-demand Distance Vector Routing (AODV) and proactive protocol based on the rule of the tree structure [9]. It works in second layer model ISOOSI and uses Radio-Aware as a metric for routing.

Thanks to this approach it is possible to create a path structure ready to be used during data transmission, thereby reducing delay associated with creating them. On the other hand, 
taking into account the variability of parameters in a wireless network environment, there is a chance to select alternative paths with better parameters at the particular time.

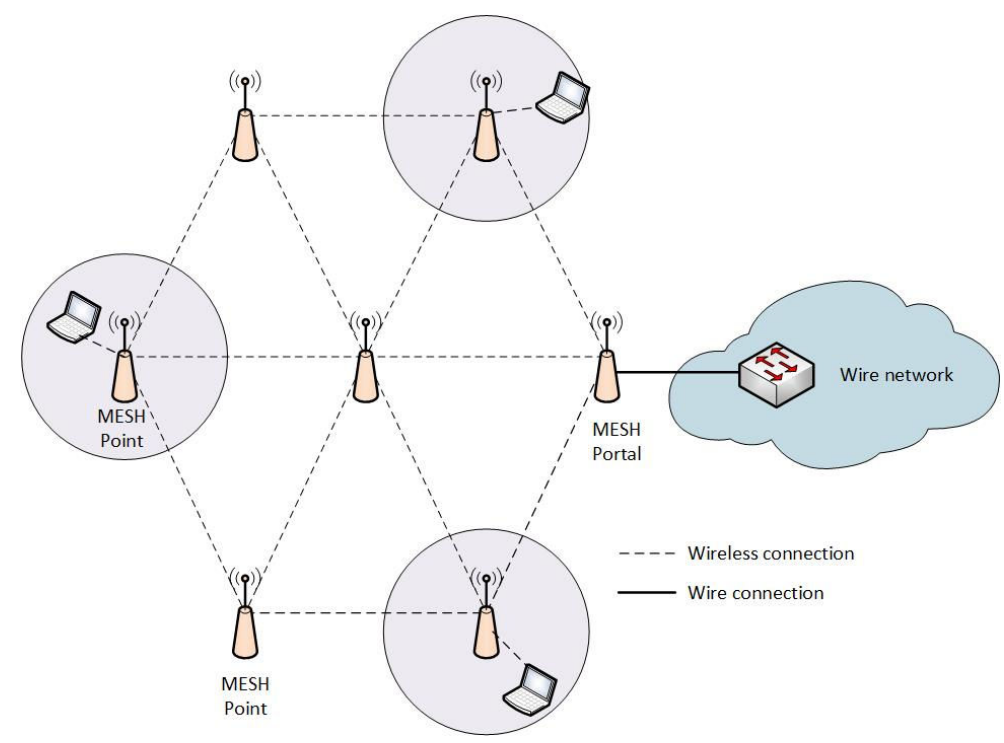

Fig. 1. Example of wireless MESH network infrastructure.

The HWMP allows to create efficient converged networks using WLAN network [10]. The traffic in the converged networks is sensitive to the variability of transmission parameters such as delay, bandwidth, packet retransmission and more. This is why the current update of information about network environment and improvement of the mechanism for choosing "better" transmission paths is the recommended process.

\section{Current metric}

Various parameters and metrics are used in protocols and routing algorithms for different computer networks. Most popular are: distance (number of hops), bandwidth, links reliability, rate of errors and more [11]. In wireless MESH networks the number of hops and a metric are the most important components in the process of selecting the best route that ensures the connection for this type of network.

The number of hops determines the number of points between a local point and the destination; however, this number is not enough to determine the best route. Sometimes, more beneficial are two fast links to compare with one that is overloaded. The metrics have a data indicator and an error rate indicator. In order to achieve better effectivity, the metrics are used instead of the number of hops. It is important to remember that a given metric can map the current situation from the point of view of a given node, rather than the entire network or transmission path. It is related to high dynamics of possible changes in parameters or interference.

IEEE $802.11 \mathrm{~s}$ describes standard metrics of connections and determines the use of optional metrics. In order to ensure a possible metric routing, all points are forced to constant actualization of the selection metric. In MESH network 'Airtime Link Metric' is used to calculate the route of each pair. It determines the number of source channels that are used to transmit frames through a specific link. Airtime $C_{a}$ is described in a category of coefficient modulation $r$ and $a$ bit error expressed by coefficient $e_{p t}$ for the frame size $B_{t}$ : 


$$
C_{a}=\left(O_{c a}+O_{p}+\frac{B_{t}}{r}\right) \frac{1}{1-e_{p t}}
$$

where $O_{c a}$ denotes channel access overhead, $O_{p}$ is a protocol overhead and $B_{t}$ are constants defined for each type of modulation 802.11 [12].

Table 1. IEEE 802.11s Airtime Link Metric constants.

\begin{tabular}{|c|c|c|c|}
\hline Parameter & $\mathbf{8 0 2 . 1 1 a}$ & $\mathbf{8 0 2 . 1 1 b}$ & Description \\
\hline$O_{\text {ca }}$ & $75 \mu \mathrm{s}$ & $335 \mu \mathrm{s}$ & channel access overhead \\
\hline$O_{\mathrm{p}}$ & $110 \mu \mathrm{s}$ & $364 \mu \mathrm{s}$ & protocol overhead \\
\hline$B_{\mathrm{t}}$ & 8192 & 8224 & number of bits in frame \\
\hline
\end{tabular}

Nowadays, the used metric is taking into account the channel access overhead, frame heading and protocol overhead, for which parameters $O_{c a}$ and $O_{p}$ are responsible. Parameter $B_{t} / r$ determines the time in which the frame will be sent. Parameter $1 /\left(1-e_{p t}\right)$ determines the reliability of the connection. In case of multicast and broadcast traffic, the error rate is not efficient as sent packets are unconfirmed. Thus, the error rate, from the point of view of a given node, will not reflect the real situation. It needs to be remembered that wireless networks are treated as converged networks in which video and audio transmission is as important (and even more important) as traditional data transmission. The MESH networks can be used to stream traffic from monitoring so that link quality and its selection will affect the level of security. Therefore, till now, apart from stable contents in metric, variable values were associated with error rate and the bandwidth of transmission channel.

\section{Proposed solution}

The proposed modification of the existing metric is based on a single transmission frequency for traffic between nodes, and the traffic between nodes and the end users.

The general principle for selecting a more beneficial metric is as following:

- $\quad$ metric $a$ is better than $b$, where $a<b$;

- $\quad$ metric $a$ is equal to metric $b$, where $a=b$;

- $\quad$ metric $a$ is worse than metric $b$, where $a>b$.

Using this rule, a modification of the existing solution for calculating of metric connection has been proposed. This modification can be presented as follows:

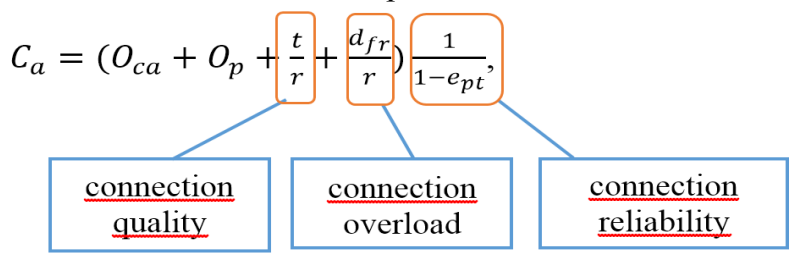

$O_{c a}$ is channel access overhead. This value is constant and depends on the IEEE 802.11 standard that is used. The values are defined in Table 1.

$O_{p}$ is a protocol overhead. This value is constant and it depends on The IEEE 802.11 standard that is used.

$t / r$ determine the quality of the connection. Parameter $t$ defines the delay in connection between a pair of nodes. Parameter $r$ defines the connection bandwidth. The value of the delay parameter should be measured as an average value in specified and constant time intervals. 
$d_{f r} / r$ determines the connection overload. Parameter $d_{f r}$ is the sum of packets received and sent. Parameter $r$ is the connection bandwidth. This value precisely determines the load of the channel from the point of view of a given node. We need to remember that during single channel transmission we are dealing with the half-duplex transmission type.

$1 /\left(1-e_{p t}\right)$ determines the reliability of the connection. Parameter $e_{p t}$ is the percentage of the error rate package.

The proposed approach, taking the quality and load of connection into account, adjusts the metric at a given time to conditions in which network operates. This solution makes it possible to predict the overload of connection in the future. This prevents the excessive use of the selected link by individual agents.

The proposed metric is considered in terms of load variability and quality. The metric changes with the change of individual parameters. The chart below Fig. 2 illustrates the relationship between the bandwidth and the total number of packets received and sent. The parameter responsible for the delay in these calculations is constant and its value is $20 \mathrm{~ms}$. Reliability of the connection is also constant and its effectiveness is $100 \%$.

By analyzing the charts in Fig. 2 and Fig. 3, metric's declining trend for both IEEE 802.11a and IEEE $802.11 \mathrm{~b}$ can be observed. This is due to the increase of the capacity for three different values responsible for the connection overload.

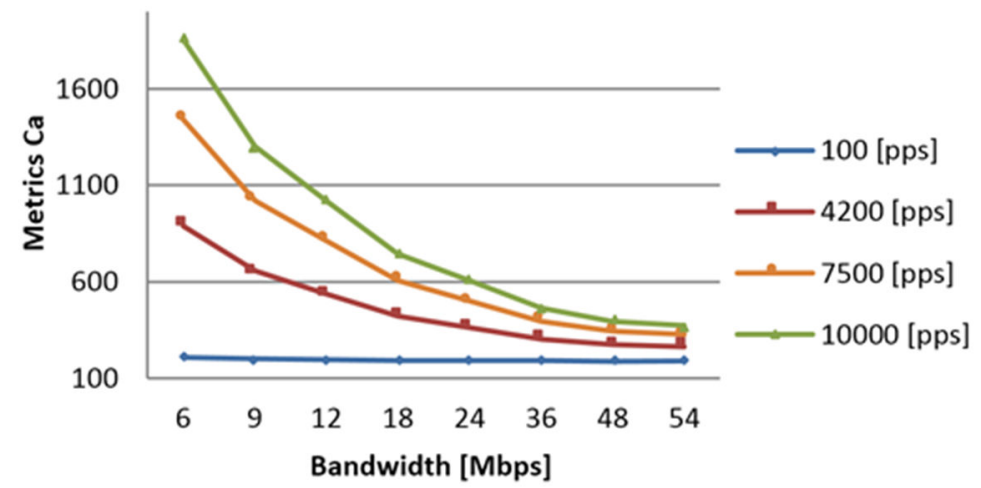

Fig. 2. Airtime link metric - IEEE 802.11a load package.

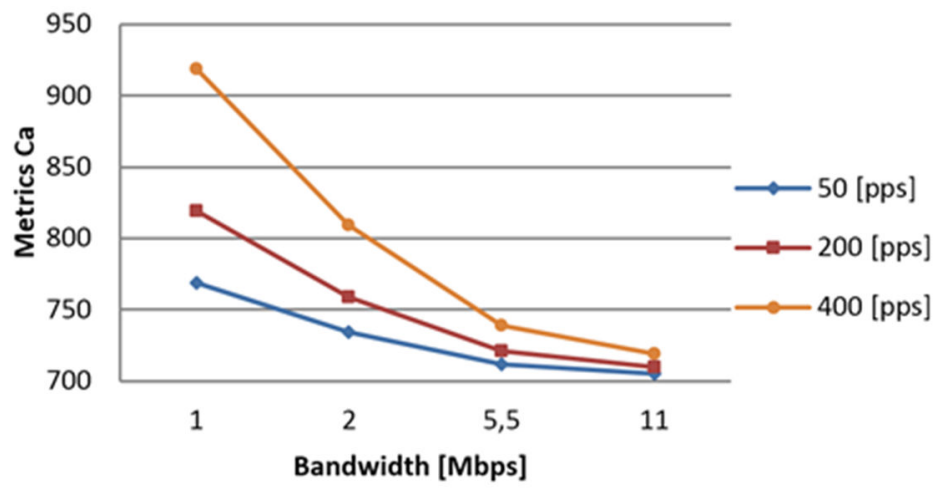

Fig. 3. Airtime link metric - IEEE $802.11 \mathrm{~b}$ load package.

The tables below: Table 2 and Table 3 include metrics value considered in a broader range of connection overload. Table 2 refers to the IEEE 802.11a standard and Table 3 contains values for the IEEE $802.11 \mathrm{~b}$ standard. These values significantly diverge from each other at a lower bandwidth connection. The important part of the proposed metric is the part responsible for the connection overload. 
Table 2. The value of the metrics depending on the load package - IEEE 802.11a.

\begin{tabular}{|c|c|c|c|c|c|}
\hline$[$ Mbps]\pps] & $\mathbf{1 0 0}$ & $\mathbf{8 0 0}$ & $\mathbf{4 2 0 0}$ & $\mathbf{7 5 0 0}$ & $\mathbf{1 0 0 0 0}$ \\
\hline $\mathbf{6}$ & 205 & 355 & 888 & 1438 & 1855 \\
\hline $\mathbf{9}$ & 198 & 298 & 654 & 1021 & 1298 \\
\hline $\mathbf{1 2}$ & 195 & 270 & 537 & 812 & 1020 \\
\hline $\mathbf{1 8}$ & 192 & 242 & 419 & 603 & 742 \\
\hline $\mathbf{2 4}$ & 190 & 228 & 361 & 498 & 603 \\
\hline $\mathbf{3 6}$ & 188 & 213 & 302 & 394 & 463 \\
\hline $\mathbf{4 8}$ & 188 & 206 & 273 & 342 & 394 \\
\hline $\mathbf{5 4}$ & 187 & 204 & 263 & 324 & 371 \\
\hline
\end{tabular}

Table 3. The value of the metrics depending on the load package - IEEE $802.11 \mathrm{~b}$.

\begin{tabular}{|c|c|c|c|c|c|}
\hline [Mbps]\[pps] & $\mathbf{5 0}$ & $\mathbf{1 0 0}$ & $\mathbf{2 0 0}$ & $\mathbf{3 0 0}$ & $\mathbf{4 0 0}$ \\
\hline $\mathbf{1}$ & 769 & 819 & 919 & 1019 & 1119 \\
\hline $\mathbf{2}$ & 734 & 759 & 809 & 859 & 909 \\
\hline $\mathbf{5 , 5}$ & 712 & 721 & 739 & 757 & 775 \\
\hline $\mathbf{1 1}$ & 705 & 710 & 719 & 728 & 737 \\
\hline
\end{tabular}

The following charts present the variability of quality. Fig. 4 refers to the IEEE $802.11 \mathrm{a}$ standard and Fig. 5 presents the value for the IEEE $802.11 \mathrm{~b}$ standard. The parameter responsible for the number of packets received and sent is constant and its value is 400pps. The parameter responsible for the reliability of the connection is also constant and its effectiveness is $100 \%$.

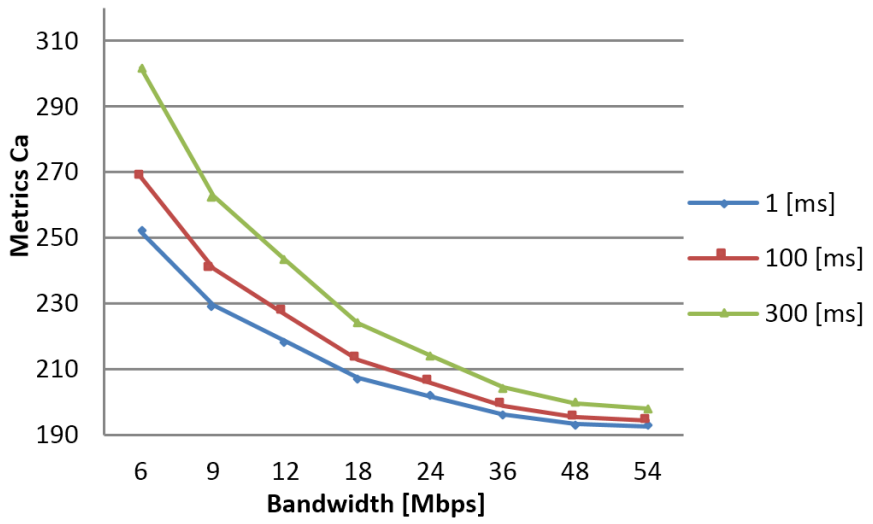

Fig. 4. Airtime link metric - IEEE 802.11a link quality. 


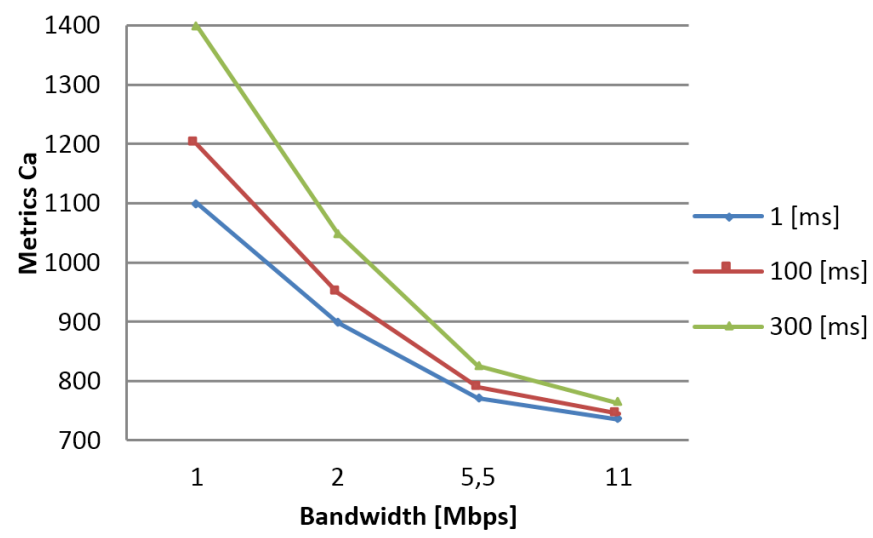

Fig. 5. Airtime link metric - IEEE $802.11 \mathrm{~b}$ link quality.

The above charts present metrics variation which depends on the bandwidth parameters and the connection delay. The tendency is also declining, resulting from the increase in bandwidth and the decline in the value responsible for the connection delay.

The following tables: Table 4 and Table 5 include values of metrics dependent on the bandwidth and connection delay. Table 4 refers to the IEEE 802.11a standard and Table 5 contains values for the IEEE $802.11 \mathrm{~b}$ standard. Here the metric was checked in a wider range of time response. Its change affects the change of the oveall value of the metric to a lesser extent than the change values responsible for the connections overload.

Table 4. The value of the metrics depending on the load time - IEEE 802.11a.

\begin{tabular}{|c|c|c|c|c|c|}
\hline$[$ Mbps $]\lceil[\mathbf{m s}]$ & $\mathbf{1}$ & $\mathbf{5 0}$ & $\mathbf{1 0 0}$ & $\mathbf{3 0 0}$ & $\mathbf{5 0 0}$ \\
\hline $\mathbf{6}$ & 252 & 260 & 268 & 302 & 335 \\
\hline $\mathbf{9}$ & 230 & 235 & 241 & 263 & 285 \\
\hline $\mathbf{1 2}$ & 218 & 223 & 227 & 243 & 260 \\
\hline $\mathbf{1 8}$ & 207 & 210 & 213 & 224 & 235 \\
\hline $\mathbf{2 4}$ & 202 & 204 & 206 & 214 & 223 \\
\hline $\mathbf{3 6}$ & 196 & 198 & 199 & 204 & 210 \\
\hline $\mathbf{4 8}$ & 193 & 194 & 195 & 200 & 204 \\
\hline $\mathbf{5 4}$ & 192 & 193 & 194 & 198 & 202 \\
\hline
\end{tabular}

Table 5. The value of the metrics depending on the load time - IEEE 802.11b.

\begin{tabular}{|c|c|c|c|c|c|}
\hline$[$ Mbps $\backslash[\mathbf{m s}]$ & $\mathbf{1}$ & $\mathbf{5 0}$ & $\mathbf{1 0 0}$ & $\mathbf{3 0 0}$ & $\mathbf{5 0 0}$ \\
\hline $\mathbf{1}$ & 1100 & 1149 & 1199 & 1399 & 1599 \\
\hline $\mathbf{2}$ & 900 & 924 & 949 & 1049 & 1149 \\
\hline $\mathbf{5 , 5}$ & 772 & 781 & 790 & 826 & 863 \\
\hline $\mathbf{1 1}$ & 735 & 740 & 744 & 763 & 781 \\
\hline
\end{tabular}


By analyzing metric used in the HWMP protocol it can be concluded that the metric does not reflect the influence of the most important properties of the link that should be used to calculate metrics. Properties such as bandwidth, load and delay in connection more precisely reflect its current status.

The following charts: Fig. 6 and Fig. 7 present Airtime metric with the metric values of the proposed solution. By analyzing data presented in the chart, it can be concluded that the proposed metric model takes the current state of the connection overload into account. Thanks to that the routing protocol will select the best path based on their decision on the lowest connection overload at that moment. This metric is in favour of the path with a lower load, not as in the case of the old metric model which takes into account the bandwidth connection as the main element of the formula

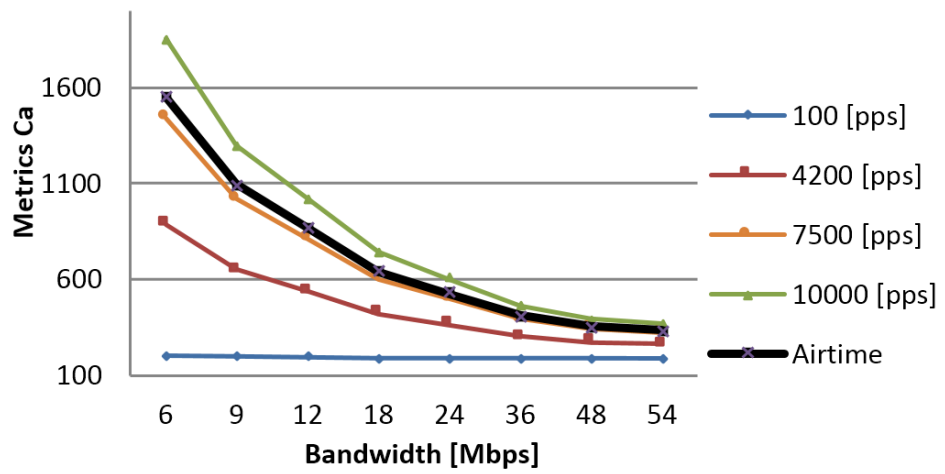

Fig. 6. Compare IEEE 802.11a Airtime link metric with load package.

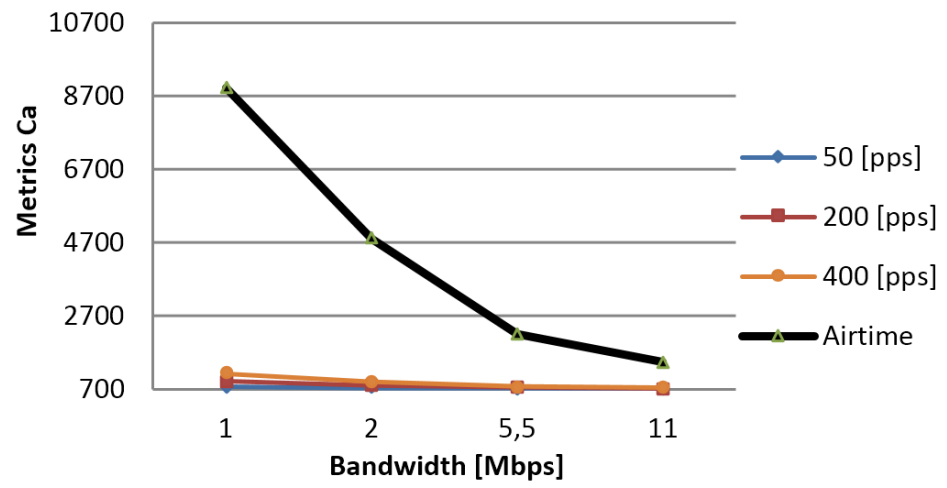

Fig. 5. Compare IEEE $802.11 \mathrm{~b}$ Airtime link metric with load package.

\section{Conclusions}

Currently, we are witnessing rapid development and increasing scope of wireless networks application. This fact is affected by many factors such as popularization of mobile devices, the development of new network services, etc. Therefore, it is necessary to search for new solutions and improve existing ones. The modification of route selection methodology for wireless MESH networks proposed in this paper allows for their better adaptation to the current and changing conditions of the network environment. It introduced parameters regarding the connection quality and overload. The obtained simulation results show a close dependence of the metric values on the bandwidth and delay parameters. The proposed metric favours connections with less overload. This approach may be important especially 
in the case of a converged network. This type of network that combines different types of network traffic, requires the use of links/paths of good quality, high reliability, lack of overload. It seems that the proposed solution takes these requirements into account.

\section{References}

1. J.K. Gimzewski, A.Z. Stieg, V. Vesna, Self-Organization and Emergence of Dynamic Systems. Handbook of Science and Technology Convergence, part I, 163-180 (2016)

2. C. Prehofer, C. Bettstetter, Self-organization in communication networks: principles and design paradigms, IEEE Communications Magazine, 43, 78-85 (2005)

3. W. Elmenreich, F. Dressler, V. Loreto, Self-Organizing Systems. Lecture Notes in Computer Science, 8221 (2014)

4. W. Banzhaf, Self-Organizing Systems. Encyclopedia of Complexity and Systems Science, Springer (2009)

5. V.V. Isaeva, Self-organization in biological systems. Biology Bulletin, 39, 110-118 (2012)

6. G.R. Hiertz, D. Denteneer, S. Max, R. Taori, J. Cardona, L. Berlemann, B. Walke, IEEE 802.11s: The WLAN Mesh Standard, IEEE Wireless Communications, 17, 104$111(2010)$

7. A. Sgora, D.D. Vergados, P. Chatzimisios, IEEE 802.11s Wireless Mesh Networks: Challenges and Perspectives. Social Informatics and Telecommunications Engineering, 13, 263-271 (2009)

8. H. Haken, Information and Self-Organization A Macroscopic Approach to Complex Systems, Springer (2006)

9. S.M.S. Bari, F. Anwar, M.H. Masud, Performance study of hybrid Wireless Mesh Protocol (HWMP) for IEEE 802.11s WLAN mesh networks, International Conference on Computer and Communication Engineering (ICCCE), 712-716 (2012)

10. A. Paszkiewicz, M. Bolanowski, P. Zapała, Convergent traffic in the environment of wireless MESH technology, Annales UMCS Sectio AI Informatica, 13, 84-95 (2013)

11. J. Guerin, M. Portmann, A. Pirzada, Routing metrics for multi-radio wireless mesh networks, Telecommunication Networks and Applications Conference, 343-348 (2007)

12. S. Misra, S.Ch. Misra, I. Zhang (Eds.), Guide to Wireless Mesh Networks, Springer (2009) 